\title{
ORIGINAL
}

\section{ESTIMACIÓN DE LOS COSTOS DE LA DETECCIÓN DE CASOS DE TUBERCULOSIS. MUNICIPIO HABANA VIEJA. 2002}

\author{
Mariana Peralta Pérez (1), Ana M. Gálvez González (2) y Edilberto González Ochoa (3) \\ (1) Grupo Provincial de Control de la Tuberculosis. Ciudad de la Habana. \\ (2) Comité académico de la Maestría en Economía de la Salud. Escuela Nacional de Salud Pública. La Habana. \\ (3) Grupo de Vigilancia e Investigaciones en TB, Lepra e IRA. "Instituto "Pedro Kourí".
}

\section{RESUMEN}

Fundamentos: El Programa de Control de Tuberculosis en Cuba ha logrado reducir significativamente la incidencia de casos en todas sus formas. Desde hace 5 años el municipio Habana Vieja ha mantenido la incidencia más alta de la provincia Ciudad de La Habana y una de las más altas del país. El objetivo del trabajo fue estimar el costo de la detección de casos de tuberculosis en el municipio Habana Vieja durante el año 2002.

Métodos: Se realizó un estudio descriptivo retrospectivo de estimación de costos, con perspectiva social. Se estimaron costos de la detección de casos y sus partidas en instituciones de salud. Para pacientes con tos/expectoración >o igual 14 días $(\mathrm{SR+14})$ se estimó el gasto de bolsillo y pérdidas monetarias por ausencias laborales. Los costos se expresaron en pesos cubanos equivalentes a dólares Americanos ( $1 \mathrm{CUC}=1$ USD). Se obtuvo información de registros oficiales en instituciones de salud y entrevistas a trabajadores y SR+14.

Resultados: En promedio el costo social de la detección de casos de tuberculosis para un SR+14 en CUC fue de 24,11 y el institucional de 12,55; la investigación clínica 0,37 ; la baciloscopía 2,25; el Cultivo 7,05; la radiografía de tórax 1,67; la notificación 3,07 ; y registro 0,36 .

Conclusiones: Los mayores costos se observaron en la realización de baciloscopías y cultivos; el salario y los reactivos fueron las partidas que más aportaron a ese costo.

Palabras clave: Tuberculosis. Evaluación económica. Costo. Programa. Detección de casos.

Correspondencia:

Edilberto González Ochoa.

Instituto "Pedro Kourí"

Autopista Novia del Mediodía Km. 6,5

Apdo. 601. Playa.

Teléfono 5372020652 ; FAX 537204605.

Correo electrónico: ochoa@ipk.sld.cu

*Este trabajo se llevó a cabo con la ayuda del Instituto de Medicina Tropical de Amberes, Bélgica.

\section{ABSTRACT}

\section{Costs Estimation of Tuberculosis \\ Cases Detection. La Habana Vieja Municipality, Cuba. 2002}

Background: The Cuban Tuberculosis Control Program has been able to significantly reduce the tuberculosis cases incidence in all its forms. La Habana Vieja municipality has maintained the highest incidence in Havana City province during 5 years and one of the highest in the country.

Objective: To estimate the cost of Tuberculosis cases detection in Habana Vieja municipality, in the year 2002.

Methods: A descriptive retrospective study to estimate the costs with social perspective was carried out. The costs of cases detection and their departures in health facilities were considered. For patients with cough/expectoration $\geq 14$ days $(\mathrm{RS}+14)$ the pocket expense and monetary losses for labour absences were considered. Costs were expressed in equivalent Cuban pesos to American dollars (1 CUC $=1$ USD). Information from official records in health institutions and from interviews to workers and RS+14 was obtained.

Results: Social cost of tuberculosis cases detection for an RS+14 was in average 24,11 CUC, and institutional cost was 12,55; for clinical investigation 0.37 ; for sputum smear microscopy 2,25 ; for culture 7,05 ; for thorax X-ray 1,67 ; for notification 3,07; and for registering 0,36 .

Conclusions: The biggest costs were observed in sputum smear microscopies and cultures performance; salaries and reagents were the issues contributing more in that cost. The results obtained in this study could be extrapolated to other municipalities in the country with social and economic conditions similar to La Habana Vieja.

Words key: Tuberculosis. Economic evaluation. Cost. Program. Cases detection. 


\section{INTRODUCCIÓN}

La detección de casos es una de las estrategias esenciales para el control de la tuberculosis ${ }^{1}$. De ella depende en un primer paso la posibilidad de un impacto efectivo con el tratamiento. En los escenarios de países o territorios depauperados económicamente es preciso obtener con urgencia una buena tasa de detección de casos de tuberculosis, para tratar de reducir la transmisión mediante la quimioterapia estándar, para lo cual la estrategia DOTS-TAES (Tratamiento acortado estrictamente supervisado) debe ser extendida ${ }^{1}$. Para lograr este objetivo es necesario un fuerte soporte económico, por lo que la estimación de los costos es un elemento importante para los tomadores de decisiones $^{2}$. Aun en países de baja prevalencia su estimación es primordial para la planificación de programas hacia la eliminación y puede servir de base para estudios de costo/efectividad que puedan contrastarse con los de otros países ${ }^{3-13}$.

La detección de casos es un proceso complejo integrado por al menos cuatro componentes: 1. Selección de los grupos e individuos con alto riesgo de presentar tuberculosis; 2. Educación para la mejor participación popular y social; 3. Diagnóstico; 4. Registro y notificación ${ }^{14}$. Estos deben funcionar simultánea y congruentemente para obtener un rendimiento adecuado. Resultando absolutamente necesaria la estimación de los costos para la evaluación de su eficiencia.

En Cuba el impacto obtenido por el Programa Nacional de Control de la Tuberculosis (PNCT) ha permitido reducir significativamente la incidencia de la enfermedad en todas sus formas por debajo de 10 por $10^{5}$ habitantes ${ }^{15-17}$. El municipio Habana Vieja, a pesar de los esfuerzos locales y provinciales para el control de la tuberculosis en ese territorio, ha mantenido la incidencia más alta de la provincia Ciudad de La Habana desde hace 5 años, según se ha reportado en los informes anuales PNCT de la provincia (datos no publicados) y una de las más altas del país. En el año 2002 la tasa de incidencia fue de 16,6 por $10^{5}$ habitantes $(1,6$ veces mayor que la de la provincia y $2,2$ veces del país $)^{18}$. Se diagnosticaron en el territorio 16 casos nuevos, de los cuales 15 presentaron localización pulmonar $(93,7 \%)$. La coinfección tuberculosis/virus de la inmunodeficiencia humana (VIH) fue de un $12,5 \%$.

Para avanzar en la eliminación de la tuberculosis como problema de salud se intenta modificar algunos elementos del PNCT. El costo del programa de control sería una herramienta útil para contribuir a la toma de estas decisiones; pero se han publicado escasos estudios de relacionados con este tema ${ }^{16,19}$.

El objetivo de este trabajo fue estimar el costo de la detección de casos de tuberculosis en el municipio Habana Vieja durante el año 2002, para ser utilizado como base en la realización de futuros estudios de costo-efectividad.

\section{SUJETOS Y MÉTODOS}

Se realizó un estudio descriptivo retrospectivo de estimación de costos (evaluación económica parcial $)^{2}$. La población objeto de estudio fueron los pacientes de 15 y más años de edad, con tos/expectoración $>\mathrm{o}=14$ días $(\mathrm{SR}+14)$ identificados en el municipio en la Habana Vieja, durante el año 2002. Ese territorio está ubicado en la zona centro-norte de la capital, con una población estimada para ese año de 96112 habitantes. Se garantizó la atención médica por 5 Áreas de Salud (policlínicas): "Diego Tamayo, Tomas Romay, Antonio Guiteras, Ángel A. Aballí Robert M. Zulueta" con poblaciones de $13.528 ; 28.278 ; 7.613 ; 27.169$ y 
19.524 habitantes respectivamente. Consta además con una unidad municipal de Higiene y epidemiología (UMHE) que asesora metodológicamente y controla algunos recursos del programa. En cada policlínica se realizaron los exámenes directos de esputos; los cultivos se realizaron en el laboratorio municipal de microbiología (LMM), ubicado fuera del territorio de estudio (municipio Plaza de la Revolución).

Para la realización de esta investigación se siguieron los pasos recomendados en la Guía Metodológica para la Evaluación Económica en Salud Cuba ${ }^{20}$. Los costos fueron construidos según lo propuesto en el método del costeo por ingredientes $^{21}$. La perspectiva analítica del estudio, abarcó toda la sociedad al considerar los costos que pudieron recaer en cualquier individuo y pretender utilizar los resultados para mejorar la calidad de vida de la población ${ }^{22}$. Los costos se estimaron en pesos cubanos equivalentes a dólares Americanos (1 CUC = 1 USD) y fueron referidos al año 2002 (no se realizaron descuentos en el tiempo). Las fuentes para la recogida de información se obtuvieron de las direcciones de las policlínicas, LMM y UMHE (instituciones de salud), departamentos de recursos humanos, estadísticas, contabilidad laboratorios y almacenes. Se realizaron entrevistas a SR+14, al personal de las áreas de salud, LMM y UMHE vinculados a la ejecución PNCT. Se obtuvieron datos de la revisión de los registros de Sintomáticos Respiratorios (modelo 18-176-1), de Baciloscopías y Cultivos (modelo 64-30), control de reactivos, modelo SNC 225 (documento donde se registra el salario devengado por el trabajador); libro submayor de activos fijos tangibles (documento en el que se registran el conjunto bienes materiales que representan la inversión de capital o patrimonio de una dependencia o entidad en las cosas usadas o aprovechadas por ella, de modo periódi- co, permanente o semi-permanente, denota inmovilización al servicio del negocio, por lo que pueden considerarse como inversiones fijas. Resultan tangibles, al ser susceptibles de ser tocados, como las edificaciones y los equipos) ${ }^{23}$. Las variables estudiadas fueron: recursos humanos, equipos y edificaciones, reactivos y otros materiales y los gastos generales.

En cada institución de salud (policlínicas, UMHE y LMM) se estimó el costo directo de: recursos humanos, a partir del salario real devengado en una hora de trabajo de cada categoría ocupacional (fue 3,$09 ; 1,43 ; 1,84$ y 0,90 CUC para médicos, enfermeras, técnicos y mensajeros respectivamente) y tiempo dedicado al PNCT. El costo de los recursos materiales: reactivos, frascos para esputo, material de oficina, se estimó partiendo de la utilización, consumo y precio de estos recursos; mientras que el costo de equipos y edificaciones se estimó a partir de la depreciación que sufren estos bienes capitales al ser utilizados y enveje$\operatorname{cer}^{24}$. Los gastos generales en cada institución (pago por consumo de agua, electricidad y gas licuado) fueron repartidos proporcionalmente a cada departamento según el consumo, por las actividades realizadas en condiciones de habituales durante el 2002. El costo indirecto en todas las instituciones, se identificó a partir de los costos de administración, servicios y mantenimiento en el año 2002; el monto obtenido por la sumatoria de estos costos fue expresado en porcentaje del total de dinero gastado por cada institución en ese período. Se obtuvo el costo institucional a partir de la sumatoria de los costos directo e indirecto. El costo no institucional se estimó del gasto de bolsillo de los pacientes, que incluyeron gastos de transportación y de medicamentos; para ello se realizó un muestreo no aleatorio por conveniencia, debido a que el muestreo probabilística ofrecía un tamaño de muestra eleva- 
do y no compensaba los gastos para interés de la investigación, ya que no se consideró tener en cuenta una estimación del error de la muestra. Éste consistió en visitar a los SR+14 en sus domicilios (los más cercanos a cada área de salud) y entrevistarlos. Fueron entrevistados un total de $268 \mathrm{SR}+14$ en todo el municipio, 97 del área A. A. Aballí, 59 del T. Romay, 19 de A. Guiteras, 65 del D. Tamayo y 28 del R. M. Zulueta. Se estimó además el valor monetario del salario que esos sintomáticos respiratorios dejaron de recibir por ausentarse del trabajo. El costo total de la detección de casos de cada actividad se estimó para las instituciones de salud. Se calcularon los costos unitarios por actividad realizada, por $\mathrm{SR}+14$ identificado y por caso nuevo notificado de tuberculosis en cualquiera de sus formas clínicas (Caso nuevo: paciente que nunca ha sido notificado ni recibido tratamiento antituberculoso o sólo lo recibió por menos de 4 semanas. Tuberculosis pulmonar con baciloscopía positiva: paciente con lesión tuberculosa en el parénquima pulmonar con un mínimo de 2 exámenes directos de esputo positivos; o con directo de esputo y cultivo positivos junto a imagen radiográfica compatible con tuberculosis pulmonar activa. Tuberculosis pulmonar con baciloscopía negativa: paciente con lesión tuberculosa en el parénquima pulmonar con un mínimo de 2 exámenes directos de esputo negativos y tiene un cultivo positivo; o presenta 2 exámenes directos de esputo negativos, con signos radiográficos compatibles con tuberculosis pulmonar activa y ausencia de respuesta a 1 semana de tratamiento con un antibiótico de amplio espectro; o paciente muy enfermo, con al menos 2 exámenes directos de esputo negativos y signos radiográficos compatibles con tuberculosis pulmonar diseminada. Tuberculosis extrapulmonar: paciente que presenta al menos un cultivo positivo en un material procedente de una localización fuera del pulmón) ${ }^{17}$.

\section{RESULTADOS}

Durante el año 2002 se detectaron 2.036 SR+14 y se notificaron al PNCT 16 casos de tuberculosis. Se realizaron las actividades que integran la detección de casos en condiciones de funcionamiento normal del programa de control.

El costo total de la detección de casos de tuberculosis, fue estimado en 49.080,09 CUC. El valor más elevado de todos los costos fue encontrado en la UMHE y el LMM con 12.703,12 CUC (25,9\%). Las áreas de salud que alcanzaron los mayores costos fueron D. Tamayo, AA. Aballí y T. Romay las con 11.899,46; 10.156,00 y 9.769,55 CUC. El costo Institucional se estimó en 25.549,37 CUC (52,1\% del costo total). Casi la mitad de ese costo se estimó a nivel de UMHE y LMM con 12.703,12. Las policlínicas D. Tamayo y A.A. Aballí alcanzaron el mayor monto dentro del total de áreas de salud con $3.506,70$ y $3.303,75$ CUC respectivamente. El costo No Institucional alcanzó 23.530,72 CUC $(47,9 \%)$ del costo total; las policlínicas D. Tamayo (9.546,32 CUC), A.A. Aballí (6.852,25 CUC) y T. Romay (6.262,85 CUC) fueron las de mayores costos. El costo promedio de la detección de casos de tuberculosis por $\mathrm{SR}+14$ fue de 24,11 CUC, el área D. Tamayo, sobrepasó esta estimación con 24,28 CUC; R. M. Zulueta y T. Romay tuvieron costos promedio elevados con 22,04 y 21,61 CUC respectivamente. Costo unitario de la detección por caso de tuberculosis notificado fue de 3.067,51 CUC. En las policlínicas D. Tamayo, T. Romay y A. A. Aballí se obtuvieron las estimaciones más elevadas con $2.974,86 ; 2.442,39$ y $2.031,20$ CUC respectivamente (Tabla 1 ).

A la estimación del costo institucional de las actividades que integran la detección de casos, el estudio baciloscópico aportó la mitad del monto con 12.944,23 CUC. El cultivo de las muestras de esputo tuvo un 
Tabla 1

Costo de la detección de casos de TB según áreas de salud. Municipio Habana Vieja. 2002

\begin{tabular}{|c|c|c|c|c|c|c|c|c|}
\hline \multirow{2}{*}{$\begin{array}{l}\text { Áreas de Salud } \\
\text { A. A. Aballí }\end{array}$} & \multirow{2}{*}{$\begin{array}{c}\begin{array}{c}\text { Costo } \\
\text { Institucional a }\end{array} \\
3.303,75 \\
\end{array}$} & \multirow{2}{*}{$\begin{array}{c}\begin{array}{c}\text { Costo No } \\
\text { institucional }\end{array} \\
6.852,25 \\
\end{array}$} & \multicolumn{2}{|c|}{ Costo Total (\%) } & \multirow{2}{*}{$\begin{array}{c}\begin{array}{c}\text { Número de } \\
\text { SR+14 }\end{array} \\
735\end{array}$} & \multirow{2}{*}{\begin{tabular}{|c}
$\begin{array}{c}\text { Costo } \\
\text { unitario por } \\
\mathbf{S R + 1 4}\end{array}$ \\
13,82 \\
\end{tabular}} & \multirow{2}{*}{$\begin{array}{c}\begin{array}{c}\text { Número de } \\
\text { casos de } \\
\text { tuberculosis }\end{array} \\
5 \\
\end{array}$} & \multirow{2}{*}{ 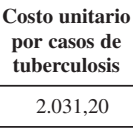 } \\
\hline & & & $10.156,00$ & $(20,7)$ & & & & \\
\hline T. Romay & $3.506,70$ & $6.262,85$ & $9.769,55$ & $(9,9)$ & 452 & 21,61 & 4 & $2.442,39$ \\
\hline A. Guiteras & $1.174,79$ & $1.677,85$ & $2.852,64$ & $(5,8)$ & 146 & 19,54 & 0 & 0.0 \\
\hline D. Tamayo & $2.353,14$ & $9.546,32$ & $11.899,46$ & $(24,2)$ & 490 & 24,28 & 4 & $2.974,86$ \\
\hline R. M. Zulueta & $2.507,87$ & $2.187,65$ & $4.695,52$ & $(9,6)$ & 213 & 22,04 & 3 & $1.565,17$ \\
\hline UMHE/LMM & $12.703,12$ & & $12.703,12$ & $(25,9)$ & 2036 & 6,24 & 16 & 793,95 \\
\hline Total & $25.549,37$ & 23.530,72 & $49.080,09$ & $(100,0)$ & 2036 & 24,11 & 16 & $3.067,51$ \\
\hline
\end{tabular}

$15 \%$ del costo institucional correspondió al costo indirecto (costo por administración, servicios y mantenimiento).

b) Costo no Institucional: Gasto de bolsillo promedio $=\$ 1,43$; por el uso de transporte $=\$ 0,27$; por utilización de medicamentos $=\$ 1,16$. Costo por ausencias al trabajo $=\$ 11,56$.

Fuentes: modelo SNC 225 (control de salarios), Registro de Depreciación de Activos Fijos Tangibles (departamentos de contabilidad); Tarjetas de

Control de medicamentos, reactivos y otros materiales (almacenes) y entrevistas a trabajadores de salud.

Tabla 2

Costo institucional de las actividades que integran la detección de casos de tuberculosis. Municipio Habana Vieja. 2002

\begin{tabular}{|c|c|c|c|c|c|}
\hline \multirow[b]{2}{*}{ Centros de salud } & \multirow{2}{*}{ Actividades } & \multicolumn{4}{|c|}{ Costo Institucional } \\
\hline & & Costo total & $\begin{array}{l}\text { Costo unitario por } \\
\text { actividad }\end{array}$ & $\begin{array}{l}\text { Costo unitario por } \\
\text { SR }+14^{\mathrm{a}}\end{array}$ & $\begin{array}{l}\text { Costo unitario por } \\
\text { caso de tuberculosis }\end{array}$ \\
\hline Pesquisa & 2.036 & 754,90 & 0,37 & 0,37 & 47,18 \\
\hline Baciloscopía & 5.752 & $12.944,23$ & 2,25 & 6,36 & 809,01 \\
\hline Cultivo & 1.234 & $8.704,78$ & 7,05 & 4,28 & 544,05 \\
\hline Radiografía de Tórax & 1.146 & $1.915,23$ & 1,67 & 0,94 & 119,70 \\
\hline Registro & 3.270 & $1.181,10$ & 0,36 & 0,58 & 73,82 \\
\hline Notificación & 16 & 49,13 & 3,07 & 0,02 & 3,07 \\
\hline Detección de casos & - & $25.549,37$ & - & 12,55 & $1.596,84$ \\
\hline
\end{tabular}

a) $\mathrm{N}=2.036$, b) $\mathrm{N}=16$

Fuentes: modelo SNC 225 (control de salarios), Registro de Depreciación de Activos Fijos Tangibles (departamentos de contabilidad); Tarjetas de

Control de medicamentos, reactivos y otros materiales (almacenes) y entrevistas a trabajadores de salud.

total de 8.704,78 CUC, el resto de las actividades tuvieron costos inferiores. El costo promedio por actividad de la realización de un cultivo, resultó ser el más elevado con 7,05 CUC. La notificación de un caso de tuberculosis al programa de control y la realización de una baciloscopía tuvieron costos elevados al compararlas con el resto de las actividades estudiadas (3,07 y 2,25 CUC). La realización de un cultivo duplicó el costo de la notificación de un caso de TB, triplicó el costo promedio de una baciloscopía, fue cuatro veces más costoso que una radiografía de tórax y costó 20 veces más que la ac- tividad de investigación clínica y registro de $\mathrm{SR}+14$. El costo unitario de la detección de casos por $\mathrm{SR}+14$ se estimó en 12,55 CUC, todas las actividades estudiadas tuvieron menos de 1 CUC en su costo promedio, a excepción de la baciloscopía y el cultivo que tuvieron costos de 6,36 y 4,28 CUC. En el costo unitario de las actividades por caso de tuberculosis notificado el examen microscópico directo de la muestra de esputo, su cultivo y la realización del estudio por radiografía del tórax alcanzaron los costos más elevados con 809,$01 ; 544,05$ y 119,70 CUC (Tabla 2). 
Tabla 3

Costo institucional de la detección de casos de tuberculosis, según partidas. Municipio Habana Vieja. 2002

\begin{tabular}{|l|c|c|c|c|}
\hline \multirow{2}{*}{ Partidas } & \multicolumn{2}{|c|}{ Costo por partida } & Costo por SR+14 & Costo por caso de tuberculosis \\
\cline { 2 - 5 } & Costo & $(\boldsymbol{\%})$ & $\mathbf{N = 2 0 3 6}$ & N=16 \\
\hline Salario & $21.101,87$ & $(82,6)$ & 10,36 & $1.318,87$ \\
\hline Reactivos & $1.648,97$ & $(6,5)$ & 0,81 & 103,06 \\
\hline Material Reutilizable & 181,54 & $(0,7)$ & 0,09 & 11,35 \\
\hline Material de Oficina & 783,31 & $(3,1)$ & 0,38 & 48,96 \\
\hline Equipos & 125,66 & $(0,5)$ & 0,06 & 7,85 \\
\hline Edificación & 489,91 & $(1,9)$ & 0,24 & 30,62 \\
\hline Gastos Generales & $1.218,12$ & $(4,8)$ & 0,60 & 76,13 \\
\hline Costo Institucional & $25.549,38$ & $(100,0)$ & 12,55 & $1.596,84$ \\
\hline
\end{tabular}

Fuentes: modelo SNC 225 (control de salarios), Registro de Depreciación de Activos Fijos Tangibles (departamentos de contabilidad); Tarjetas de Control de medicamentos, reactivos y otros materiales (almacenes) y entrevistas a trabajadores de salud.

Dentro del costo por partidas de la detección de casos de tuberculosis, el salario fue la partida que alcanzó el mayor monto, con 21.101,87 CUC (82,6\%). Los reactivos alcanzaron 1.648,97 CUC $(6,5 \%)$ y los gastos generales $1.218,12$ CUC (4,8\%). Para el resto de las partidas se estimaron costos bajos. De los 12,55 CUC que se gastaron en promedio para la detección de un $\mathrm{SR}+14$, las partidas más importantes en cuanto al monto alcanzado fueron el salario y reactivos con 10,36 CUC y 81 centavos respectivamente. Para detectar un caso de tuberculosis fue necesario invertir en promedio $1.318,87$ CUC en salario y 103,06 CUC en reactivos. (Tabla 3 ).

\section{DISCUSIÓN}

El estudio aportó datos sobre el costo social de la detección de casos de tuberculosis, estimándose en promedio por $\mathrm{SR}+14$ en $24,11 \mathrm{CUC}$, y por caso diagnosticado en 3.067,51 CUC. El costo institucional de un cultivo fue 7,05 CUC, el de una baciloscopía 2,25 CUC. La partida salario aportó más del $82 \%$ al costo en las instituciones de salud. Todas estas estimaciones han sido reportadas por primera vez en el país y resultan de sumo interés para el PNCT. Los costos son elementos particulares en el lugar y el tiempo que se producen; el haberlos estimado para la detección de casos de tuberculosis, pudiera contribuir a la adopción de decisiones en el territorio de la Habana Vieja. La descripción acerca de este elemento del PNCT pudiera servir como base para estudios de costo/efectividad que puedan contrastarse con los de otros territorios en la provincia y el país.

En general los datos obtenidos se consideraron aceptablemente válidos y confiables atendiendo a su proceso de generación y recolección. No se obtuvo información pormenorizada respecto a la cantidad de horas perdidas en la jornada laboral de $\mathrm{SR}+14$, ya fuese necesidad de guardar reposo durante la enfermedad, ni por el número y/o duración de consultas médicas a las que asistieron durante el episodio; por lo que la similitud observada entre el costo institucional y el no institucional no pudo ser analizada en detalle.

El alto costo de la detección de casos estimado en UMHE y el LMM pudiera relacionarse con que las actividades que fueron realizadas en estas instituciones 
tuvieron mayor complejidad, requirieron de más tiempo y recursos en su ejecución (los cultivos y el control de calidad) y de personal más calificado que devengaron salarios más elevados. Para las áreas salud D. Tamayo, A. A. Aballí y T. Romay se observó que más de la mitad del costo fue no institucional y dentro de él las pérdidas por ausencias laborales tuvieron el mayor peso; éste particular comportamiento del costo pudiera ser explicado porque dentro de los $\mathrm{SR}+14$ que fueron entrevistados, había un mayor número de trabajadores que en las áreas restantes, aunque también pudo haber influido el hecho de que realmente haya ocurrido un mayor número de ausencias labores entre estos pacientes; o que las ausencias labores hayan sido más prolongadas.

Actividades como el estudio baciloscópico de la muestra de esputo y la realización del cultivo, alcanzaron los mayores montos, debido al volumen de actividades de este tipo que fueron realizadas. Llama la atención el elevado número de baciloscopías (360) y cultivos (77) realizados para diagnosticar un caso, por lo que deberían ser analizados los factores que influyeron en la eficiencia de la detección de casos de tuberculosis durante el período estudiado.

En un estudio realizado en Malawi, se encontró una estructura de los costos similar a la obtenida en nuestra investigación, teniendo en cuenta para esta comparación el tiempo de realización del estudio (para la baciloscopía fue de $\$ 3,92 ; \$ 6,20$ para el cultivo y $\$ 2,82$ para la radiografía de tórax) ${ }^{10}$. Jones TF, Schaffner W. estimaron un costo promedio de la radiografía de tórax superior al de este estudio $(\$ 6,60$ por recluso $)^{13}$. Costos inferiores a los estimados en el municipio Habana Vieja, fueron obtenidos en el estudio realizado en el Centro Provincial de Ciudad de la Habana ${ }^{19}$ y el de "Costo y resultados en térmi- nos de salud del Programa de Control de la Tuberculosis en el área rural del municipio Venezuela de Ciego de Ávila en 19982001" (datos no publicados).

La variable salario, aportó a la detección de casos la mayoría del costo estimado, resultados similares se han obtenido en investigaciones relacionadas con diferentes programas de salud tales como: "Costos de la Atención Prenatal del Policlínico Docente Ramón González Coro, 1997"; "Costos y Beneficios del ingreso en el hogar, en el Policlínico Docente 26 de Julio, 1997"; "Costo social del programa de Control de la Sífilis en Regla, 1995-1996"; "Evaluación del Sistema de Vigilancia de la Sífilis Congénita en La Lisa, 1996" (datos no publicados). El costo por consumo de reactivos y los gastos generales estuvieron relacionados con el volumen de actividades realizadas precisamente a expensas de baciloscopías y cultivos.

El PNCT cubano incluye como estrategia a todos los casos de tuberculosis pulmonar y extrapulmonar y dentro de los casos de tuberculosis pulmonar a los que tienen baciloscopía positiva y baciloscopía negativa. El gasto en la ejecución del programa de control ha tenido un beneficio claro, ya que la tuberculosis en Cuba después de mantener estas estrategias desde el año 1962, ha sido disminuida su incidencia hasta la cifra de 6.5 por $10^{5}$ habitantes; una de las cifras más bajas de América, comparable además con las más bajas de Europa. Por otra parte existe el beneficio social al evitar gastos por menos enfermos, tratamientos y el mantenimiento de hospitales antituberculosos. Esto hace pensar que los costos hallados en este estudio se justifican ${ }^{15-16}$.

En el proceso de detección casos de tuberculosis en el municipio Habana Vieja durante el año 2002, se observaron los mayores costos para las partidas salario y 
reactivo, siendo la realización de baciloscopías y cultivos las actividades más costosas. Los resultados obtenidos en este estudio pudieran ser extrapolados a otros municipios del país con condiciones sociales y económicas similares a la Habana Vieja.

\section{AGRADECIMIENTOS}

Dra. Miriam Bonne Carcacés, Lic. Lilia Esther Valdés Valera, Enfermeras Angelina Estrada Bongo y Milay Robaina Hernández.

\section{BIBLIOGRAFÍA}

1. World Health Organization. Global Tuberculosis Control. Surveillance, Planning, Financing. WHO Report 2004. Geneva; 2005.

2. Drummond MF, Stoddard GL, Torrance GW. Métodos para la evaluación de los programas de atención de la salud. Madrid: Díaz Santos; 1991.

3. Organización Panamericana de la Salud. Programa Nacional de tuberculosis. Informe final 3ra Reunión Subregional de países de baja prevalencia de tuberculosis. Santiago de Chile. 11-12 Noviembre; 2003.

4. Walker D, McNerney R, Kimankinda MM, Foster S, Tihon V, Godfrey-Faussett P. An incremental cost- effectiveness analysis of the first, second and third sputum examination in the diagnosis of pulmonary tuberculosis. Int J Tuberc Lung Dis 2000; 4 (3): 246-51.

5. Floyd K. Cost and effectiveness the impact of economic studies on tuberculosis control. Tuberculosis 2003; 83(1): 187-200.

6. Nganda b, Wang'ombe J, Floyd K, Kangangi. Cost and cost- effectiveness of increased community and primary care facility involvement in tuberculosis care in Machakos District, Kenya. Int J Tuberc Lung Dis 2003; 7 (9): 14-20.

7. Sinanovic E, Floyd K, Dudley L, Azevedo V, Grant R, Maher D. Cost and cost- effectiveness community-based care for tuberculosis in Cape Town, South Africa.. Int J Tuberc Lung Dis 2003; 7 (9): 56-62.
8. Okello D, Floyd K, Adatu F, Odeke R, Gargioni G. Cost and cost- effectiveness of increased community- based care for tuberculosis patients in rural Uganda. Int J Tuberc Lung Dis 2003; 7 (9): 72-9.

9. Moalosi G, Floyd K, Phatshwane, Moeti T, Binkin N, Kenyon T. Cost and cost- effectiveness of home-based care versus hospital care for chronically ill tuberculosis patients, Francis town. Botswana. Int J Tuberc Lung Dis 2003; 7 (9): 805 .

10. Floyd K, Skeva J, Nyirenda T, Gausi F, Salaniponi F. Cost and cost- effectiveness of increased community- facility involvement in tuberculosis patients in Lilongwe District, Malawi. Int J Tuberc Lung Dis 2003; 7 (9): 29-37.

11. Schwartzman K, Menzies D. Tuberculosis Screening of Immigrants to Low-Prevalence Countries. A Cost-effectiveness Analysis. Am J Respir Crit Care Med. 2000;161 (3): 780-9.

12. Harries AD, Kwanjana JH, Hargreaves NJ, et al. Recourses for controlling tuberculosis in Malawi. Bull World Health Organ 2001; 79 (4): 32936.

13. Jones TF, Schaffiner W. Miniature chest radiograph screening for tuberculosis in jails: a costeffectiveness analysis. AM J Respir Crit Care Med 2001; 164 (1): 77-81.

14. González E y Armas L. Una propuesta de indicador sintético para valorar la intensidad y calidad de la localización de casos de tuberculosis. Bol Epidem Sem IPK. 2002; 12 (32): 256-9.

15. González E, De Armas L, Baly A, Gálvez AM, Álvarez M, Gustavo F, et al. Impacto económicosocial del programa nacional de control de la Tuberculosis (PNCT) en la población cubana. Cad Saúde Publica. Río de Janeiro. 2000; 16(23): 697-99.

16. Marrero A, Caminero JA, Rodríguez R, Bello NE. Towards elimination of Tuberculosis in a low income countries. The experience of Cuba 19621997. Thorax 2000; 55: 30-45.

17. Ministerio de Salud Pública. Programa Nacional de Control de la Tuberculosis. Manual de Normas y Procedimientos. Ciudad de la Habana: Editorial Ciencias Médicas; 1999.

18. Ministerio de Salud Pública. Dirección Nacional de Estadística. Anuario Estadístico de Salud. [Citado 15 de ene.2007] Disponible en: http://www.infomed.sld.cu/servicios/estadisticas/anuario.html. 
19. Peralta Pérez, M, Padilla González, CM., Fuentes Díaz, M y Lazo Alvarez MA. Costo del 20. Programa Nacional de Control de la Tuberculosis. Centro Provincial de Higiene y Epidemiología de Ciudad de La Habana, 2002. Rev Cubana Med Trop. 2006;.58:0.

20. Gálvez González AM. Guía Metodológica para la Evaluación Económica en Salud Cuba. 2003. Rev Cub SP. 2004: 1.
21. Creese A, Parker D. Cost analysis in Primary Health: a training manual for programme manager. Geneva: World health Organization; 1994.

22. Rovira Foros J. Glosario de términos y conceptos de uso frecuente en la evaluación económica de medicamentos y programas sanitarios. España: Química farmacéutica Bayer SA; 2000.

23. Economía: Glosario. [Citado 19 de dic.2006]. Disponible en:http://www.monografias.com/trabajos/econoglos/econoglos.shtml. 